\title{
The Fireball Shock Model of Gamma Ray Bursts
}

\author{
P. Mészáros ${ }^{1,2,3}$ \\ ${ }^{1}$ Pennsylvania State University, 525 Davey, University Park, PA 16802 \\ ${ }^{2}$ California Institute of Technology, MS 105-24, Pasadena, CA 91125 \\ ${ }^{3}$ E-mail address: nnp@astro.psu.edu
}

\begin{abstract}
Gamma-ray bursts are thought to be the outcome of a cataclysmic event leading to a relativistically expanding fireball, in which particles are accelerated at shocks and produce nonthermal radiation. We discuss the theoretical predictions of the fireball shock model and its general agreement with observations. Some of the recent work deals with the collimation of the outflow and its implications for the energetics, the production of prompt bright flashes at wavelenghts much longer than gamma-rays, the time structure of the afterglow, its dependence on the central engine or progenitor system behavior, and the role of the environment on the evolution of the afterglow.
\end{abstract}

\section{INTRODUCTION}

Gamma-ray bursts (GRB) have been studied in gamma-rays for over 25 years, but except for rare and fleeting X-ray detections, until a few years ago there existed no longer-lasting detections at softer wavelengths. However in early 1997 the Italian-Dutch satellite Beppo-SAX suceeded in providing accurate X-ray locations and images that allowed their follow-up with large ground-based optical and radio telescopes. The current interpretation of the gamma-ray and longer wavelength radiation is that the progenitor trigger produces an expanding relativistic fireball which can undergo both internal shocks leading to gamma-rays, and (as it decelerates on the external medium) an external blast wave and a reverse shock producing a broad-band spectrum lasting much longer.

A strong confirmation of the generic fireball shock model came from the correct prediction [43], in advance of the observations, of the quantitative nature of afterglows at longer wavelengths, in substantial agreement with the subsequent data $[89,85,91,73,96]$. The measured $\gamma$-ray fluences imply a total energy of order $10^{54}\left(\Omega_{\gamma} / 4 \pi\right)$ ergs, where $\Delta \Omega_{\gamma}$ is the solid angle into which the gamma-rays are beamed. Collimation may indeed be present, evidence having been recently

CP522, Cosmic Explosions: Tenth Astrophysical Conference, edited by Stephen S. Holt and William W. Zhang

(c) 2000 American Institute of Physics 1-56396-943-2/00/\$17.00

213 
reported for this $[34,18,12]$. In any case, such energies are possible [44] in the context of compact mergers involving neutron star-neutron star (NS-NS) or black hole-neutron star (BH-NS ) binaries, or in hypernova/collapsar models involving a massive stellar progenitor $[56,68]$. In both cases, one is led to rely on MHD extraction of the spin energy of a disrupted torus and/or a central BH to power a relativistic outflow.

\section{THE GENERIC FIREBALL SHOCK SCENARIO}

Whatever the GRB trigger is, the ultimate result must unavoidably be an $e^{ \pm}, \gamma$ fireball, which is initially optically thick. The initial dimensions must be of order $r_{\text {min }} \lesssim c t_{\text {var }} \sim 10^{7} \mathrm{~cm}$, since variability timescales are $t_{\text {var }} \lesssim 10^{-3} \mathrm{~s}$. Most of the spectral energy is observed above $0.5 \mathrm{MeV}$, hence the $\gamma \gamma \rightarrow e^{ \pm}$mean free path is very short. Many bursts show spectra extending above $1 \mathrm{GeV}$, indicating the presence of a mechanism which avoids degrading these via photon-photon interactions to energies below the threshold $m_{e} c^{2}=0.511 \mathrm{MeV}$. The inference is that the flow must be expanding with a very high Lorentz factor $\Gamma$, since then the relative angle at which the photons collide is less than $\Gamma^{-1}$ and the threshold for the pair production is diminished [25]. However, the observed $\gamma$-ray spectrum is generally a broken power law, i.e., highly nonthermal. In addition, the expansion would lead to a conversion of internal into kinetic energy, so even after the fireball becomes optically thin, it would be radiatively inefficient, most of the energy being kinetic, rather than in photons.

The simplest way to achieve high efficiency and a nonthermal spectrum is by reconverting the kinetic energy of the flow into random energy via shocks after the flow has become optically thin [70]. Two different types of shocks may arise in this scenario. In the first case (a) the expanding fireball runs into an external medium (the ISM, or a pre-ejected stellar wind $[70,39,30,78]$. The second possibility (b) is that $[71,54]$, even before external shocks occur, internal shocks develop in the relativistic wind itself, faster portions of the flow catching up with the slower portions. This is a generic model, which is independent of the specific nature of the progenitor.

External shocks will occur in an impulsive outflow of total energy $E_{o}$ in an external medium of average particle density $n_{o}$ at a radius $r_{d e c} \sim$ $\left(3 E_{o} / 4 \pi n_{o} m_{p} c^{2} \eta^{2}\right)^{1 / 3} \sim 10^{17} E_{53}^{1 / 3} n_{o}^{-1 / 3} \eta_{2}^{-2 / 3} \mathrm{~cm}$, and on a timescale $t_{\text {dec }} \sim$ $r_{\text {dec }} /\left(c \Gamma^{2}\right) \sim 3 \times 10^{2} E_{53}^{1 / 3} n_{o}^{-1 / 3} \eta_{2}^{-8 / 3} \mathrm{~s}$, where $\eta=\Gamma=10^{2} \eta_{2}$ is the final bulk Lorentz factor of the ejecta. Variability on timescales shorter than $t_{d e c}$ may occur on the cooling timescale or on the dynamic timescale for inhomogeneities in the external medium, but generally this is not ideal for reproducing highly variable profiles [79] (see however [15]). However, it can reproduce bursts with several peaks [58] and may therefore be applicable to the class of long, smooth bursts.

In a wind outflow [55], one assumes that a lab-frame luminosity $L_{o}$ and mass outflow $\dot{M}_{o}$ are injected at $r \sim r_{l}$ and continuously maintained over a time $t_{w}$; 
here $\eta=L_{o} / \dot{M}_{o} c^{2}$. In such wind model, internal shocks will occur at a radius [71] $r_{\text {dis }} \sim c t_{\text {var }} \eta^{2} \sim 3 \times 10^{14} t_{\text {var }} \eta_{2}^{2} \mathrm{~cm}$, on a timescale $t_{w} \gg t_{\text {var }} \sim r_{\text {dis }} /\left(c \eta^{2}\right)$ s, where shells of different energies $\Delta \eta \sim \eta$ initially separated by $c t_{v}$ (where $t_{v} \leq t_{w}$ is the timescale of typical variations in the energy at $r_{l}$ ) catch up with each other. In order for internal shocks to occur above the wind photosphere $r_{p h} \sim \dot{M} \sigma_{T} /\left(4 \pi m_{p} c \Gamma^{2}\right)$ $=1.2 \times 10^{14} L_{53} \eta_{2}^{-3} \mathrm{~cm}$, but also at radii greater than the saturation radius (so the bulk of the energy does not appear in the photospheric quasi-thermal component) one needs to have $7.5 \times 10^{1} L_{51}^{1 / 5} t_{\text {var }}^{-1 / 5} \lesssim \eta 3 \times 10^{2} L_{53}^{1 / 4} t_{\text {var }}^{-1 / 4}$. This type of models have the advantage [71] that they allow an arbitrarily complicated light curve, the shortest variation timescale $t_{v a r} \gtrsim 10^{-3} \mathrm{~s}$ being limited only by the dynamic timescale at $r_{l}$, where the energy input may be expected to vary chaotically. Such internal shocks have been shown explicitly to reproduce (and be required by) some of the more complicated light curves [79,33,61].

A potentially valuable diagnostic tool for the central engine of GRB is the power density spectrum (PDS). An analysis of BATSE light curves [3] indicates that the logarithmic slope of the PDS between $10^{-2}$ and $2 \mathrm{~Hz}$ is approximately $-5 / 3$, and there is a cutoff of the average PDS above $2 \mathrm{~Hz}$. Using a simple kinematical

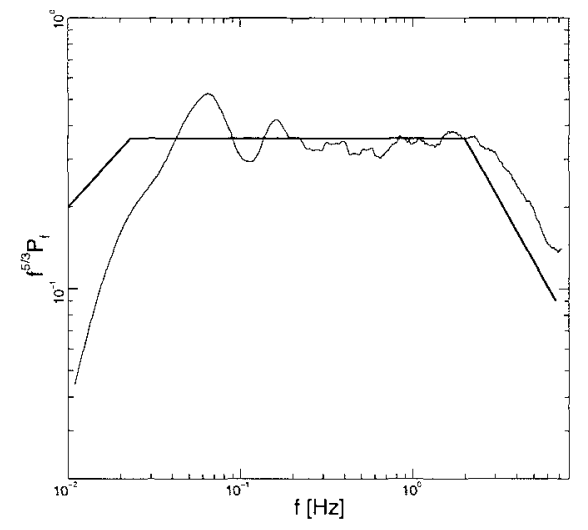

FIGURE 1. Average power density spectrum $P_{f}$ of simulated bursts from internal shocks, compared with the observed PDS (thick line), using a square-sine modulated Lorentz factor and a cosmological distribution satisfying the observed $\operatorname{logN}-\log \mathrm{P}$ (Spada, Panaitescu \& Mészáros 1999).

model for the ejection and collision of relativistic shells $[63,83]$ have calculated the light curves and PDS expected for a range of total burst energies and for a total mass ejected and bulk Lorentz factor distribution compatible with the internal shock scenario (Figure 1). The redshift distribution also affects the PDS, and the observed $\log \mathrm{N}-\log \mathrm{P}$ relation is used as a constraint. For optically thin winds, a slope approaching $-5 / 3$ requires a non-random Lorentz factor distribution, e.g. with an asymmetrical time modulation so as to produce a larger number of collisions at low frequencies (see also [4]). A cutoff at high frequencies $(\sim 2 \mathrm{~Hz})$ can be understood in terms of shocks which increasingly occur below the scattering photosphere of the outflow, or a deficit of energy in short pulses due to the modulation of the Lorentz factors favoring shocks arising further out.

A significant fraction of bursts appear to have low energy spectral slopes steeper 
than $1 / 3$ in energy $[69,14]$. This has motivated consideration of a thermal or nonthermal $[35,36]$ comptonization mechanism, which can be put in the astrophysical context [23] of internal shocks leading to self-regulated pair formation. There is also
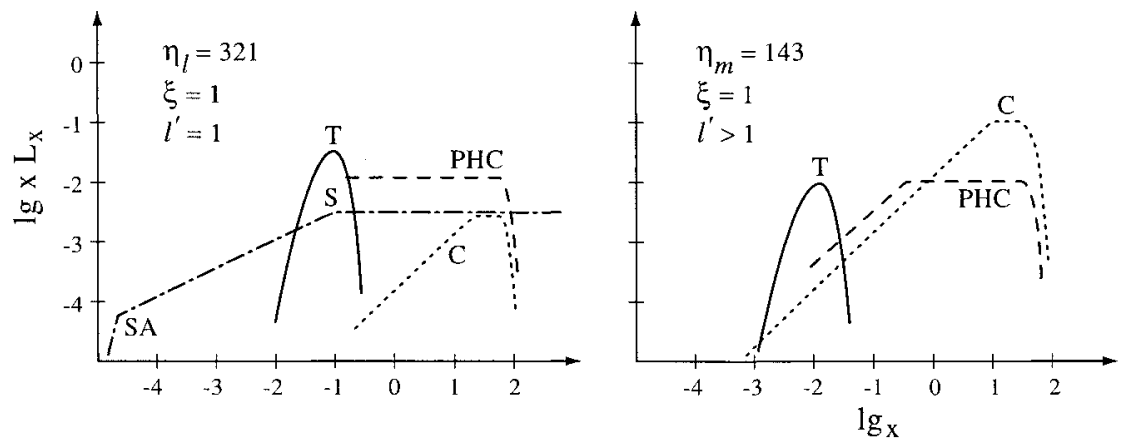

FIGURE 2. Luminosity per decade $x L_{x}$ vs. $x=h \nu / m_{e} c^{2}$ for two values of $\eta=L / \dot{M} c^{2}$ and marginal (left) or large (rigt) pair compactness. T: thermal photosphere, PHC: photospheric comptonized component; S: shock synchrotron; C: shock pair dominated comptonized component (Mészáros \& Rees, 1999b).

evidence that the clustering of the break energy of GRB spectra in the $50-500 \mathrm{keV}$ range may not be due to observational selection $[69,10,16]$. Models using Compton attenuation [11] require reprocessing by an external medium whose column density adjusts itself to a few $\mathrm{g} \mathrm{cm}^{-2}$. More recently a preferred break has been attributed to a blackbody peak at the comoving pair recombination temperature in the fireball photosphere [17]. steep low energy spectral slope being due to the Rayleigh-Jeans part of the photosphere. In order for such photospheres to occur at the pair recombination temperature in the accelerating regime requires an extremely low baryon load. For very large baryon loads, a related explanation has been invoked [87], considering scattering of photospheric photons off MHD turbulence in the coasting portion of the outflow, which upscatters the adiabatically cooled photons up to the observed break energy. These ideas have been synthesized [50] to produce a generic scenario (see Figure 2) in which the presence of a photospheric component as well as shocks subject to pair breakdown can produce steep low energy spectra and preferred breaks.

\section{SIMPLE STANDARD AFTERGLOW MODEL}

The dynamics of GRB and their afterglows can be understood independently of any uncertainties about the progenitor systems, using a generalization of the method used to model supernova remnants. The simplest hypothesis is that the afterglow is due to a relativistic expanding blast wave, which decelerates as time 
goes on [43]. The complex time structure of some bursts suggests that the central trigger may continue for up to 100 seconds, the $\gamma$-rays possibly being due to internal shocks. However, at much later times all memory of the initial time structure would be lost: essentially all that matters is how much energy and momentum has been injected; the injection can be regarded as instantaneous in the context of the afterglow. As pointed in [70], the external shock bolometric luminosity builds up as $L \propto t^{2}$ and decays as $L \propto t^{-(1+q)}$. Beyond the deceleration radius the bulk Lorentz factor decreases as a power law in radius, $\Gamma \propto r^{-g} \propto t^{-g /(1+2 g)}, r \propto t^{1 /(1+2 g)}$, with $g=(3,3 / 2)$ for the radiative or adiabatic regime (in which $\rho r^{3} \Gamma \sim$ constant or $\rho r^{3} \Gamma^{2} \sim$ constant $)$.

The synchrotron peak frequency in the observer frame is $\nu_{m} \propto \Gamma B^{\prime} \gamma^{2}$, and both the comoving field $B^{\prime}$ and electron Lorentz factor $\gamma$ are expected to be proportional to $\Gamma$ [39]. As $\Gamma$ decreases, so will $\nu_{m}$, and the radiation will move to longer wavelengths. For the forward blast wave, [53,31] discussed the possibility of detecting at late times a radio or optical afterglow of the GRB. A more detailed treatment of the fireball dynamics indicates that approximately equal amounts of energy are radiated by the forward blast wave, moving with $\sim \Gamma$ into the surrounding medium, and by a reverse shock propagating with $\Gamma_{r}-1 \sim 1$ back into the ejecta [39]. The electrons in the forward shock are hotter by a factor $\Gamma$ than in the reverse shock, producing two synchrotron peaks separated by $\Gamma^{2}$, one peak being initially in the optical (reverse) and the other in the $\gamma / X$ band (forward) $[41,42]$. Detailed calculations and predictions of the time evolution of such a forward and reverse shock afterglow model ( [43]) preceded the observations of the first afterglow GRB970228 $([13,88])$, which was detected in $\gamma$-rays, $\mathrm{X}$-rays and several optical bands, and was followed up for a number of months.

The simplest spherical afterglow model concentrates on the forward blast wave only. For this, the flux at a given frequency and the synchrotron peak frequency decay at a rate $[43,49]$

$$
F_{\nu} \propto t^{[3-2 g(1-2 \beta)] /(1+2 g)} \quad, \quad \nu_{m} \propto t^{-4 g /(1+2 g)},
$$

where $g$ is the exponent of $\Gamma \propto r^{-g}$ and $\beta$ is the photon spectral energy slope. The decay rate of the forward shock $F_{\nu}$ in equ.(1) is typically slower than that of the reverse shock [43], and the reason why the "simplest" model was stripped down to its forward shock component only is that, for the first two years 19971998, afterglows were followed in more detail only after the several hours needed by Beppo-SAX to acquire accurate positions, by which time both reverse external shock and internal shock components are expected to have become unobservable. This simple standard model has been remarkably successful at explaining the gross features and light curves of GRB 970228, GRB 970508 (after 2 days; for early rise, see $\S \mathrm{IV})$ e.g. $[96,85,91,73]$.

This simplest afterglow model has a three-segment power law spectrum with two breaks. At low frequencies there is a steeply rising synchrotron self-absorbed spectrum up to a self-absorption break $\nu_{a}$, followed by a $+1 / 3$ energy index spectrum 
up to the synchrotron break $\nu_{m}$ corresponding to the minimum energy $\gamma_{m}$ of the power-law accelerated electrons, and then a $-(p-1) / 2$ energy spectrum above this break, for electrons in the adiabatic regime (where $\gamma^{-p}$ is the electron energy distribution above $\gamma_{m}$ ). A fourth segment and a third break is expected at energies where the electron cooling time becomes short compared to the expansion time, with a spectral slope $-p / 2$ above that. With this third "cooling" break $\nu_{b}$, first calculated in [47] and more explicitly detailed in [80], one has what has come to be called the simple "standard" model of GRB afterglows. One of the predictions of this model [43] is that the relation between the temporal decay index $\alpha$, for $g=3 / 2$ in $\Gamma \propto r^{-g}$, is related to the photon spectral energy index $\beta$ through $F_{\nu} \propto t^{\alpha} \nu^{\beta}$, with $\alpha=(3 / 2) \beta$. This relationship appears to be valid in many (although not all) cases, especially after the first few days, and is compatible with an electron spectral index $p \sim 2.2-2.5$ which is typical of shock acceleration, e.g. $[91,80,95]$, etc. As the remnant expands the photon spectrum moves to lower frequencies, and the flux in a given band decays as a power law in time, whose index can change as breaks move through it. For the simple standard model, snapshot overall spectra have been deduced by extrapolating spectra at different wavebands and times using assumed simple time dependences [92,95]. These can be used to derive rough fits for the different physical parameters of the burst and environment, e.g. the total energy $E$, the magnetic and electron-proton coupling parameters $\epsilon_{B}$ and $\epsilon_{e}$ and the external density $n_{o}$.

\section{IV "POST-STANDARD" AFTERGLOW MODELS}

The most obvious departure from the simplest standard model occurs if the external medium is inhomogeneous: for instance, for $n_{e x t} \propto r^{-d}$, the energy conservation condition is $\Gamma^{2} r^{3-d} \sim$ constant, which changes significantly the temporal decay rates [47]. Such a power law dependence is expected if the external medium is a wind, say from an evolved progenitor star as implied in the hypernova scenario (such winds are generally used to fit supernova remnant models). Another departure from a simple impulsive injection approximation is obtained if the mass and energy injected during the burst duration $t_{w}$ (say tens of seconds) obeys $M(>\Gamma) \propto \Gamma^{-s}$, $E(>\Gamma) \propto \Gamma^{1-s}$, i.e. more energy emitted with lower Lorentz factors at later times (but still shorter than the gamma-ray pulse duration). This would drastically change the temporal decay rate and extend the afterglow lifetime in the relativistic regime, providing a late "energy refreshment" to the blast wave on time scales comparable to the afterglow time scale [72]. These two cases lead to a decay rate

$$
\Gamma \propto r^{-g} \propto \begin{cases}r^{-(3-d) / 2} & ; n_{e x t} \propto r^{-d} \\ r^{-3 /(1+s)} & ; E(>\Gamma) \propto \Gamma^{1-s}\end{cases}
$$

Expressions for the temporal decay index $\alpha(\beta, s, d)$ in $F_{\nu} \propto t^{\alpha}$ are given by [47,72], which now depend also on $s$ and/or $d$. The result is that the decay can be flatter (or 
steeper, depending on $s$ and $d)$ than the simple standard $\alpha=(3 / 2) \beta$. A third nonstandard effect, which is entirely natural, occurs when the energy and/or the bulk Lorentz factor injected are some function of the angle. A simple case is $E_{o} \propto \theta^{-j}$, $\Gamma_{o} \propto \theta^{-k}$ within a range of angles; this leads to the outflow at different angles shocking at different radii and its radiation arriving at the observed at different delayed times, and it has a marked effect on the time dependence of the afterglow [47], with $\alpha=\alpha(\beta, j, k)$ flatter or steeper than the standard value, depending on $j, k$. Thus in general, a temporal decay index which is a function of more than one parameter

$$
F_{\nu} \propto t^{\alpha} \nu^{\beta}, \text { with } \alpha=\alpha(\beta, d, s, j, k, \cdots)
$$

is not surprising; what is more remarkable is that, often, the simple relation $\alpha=$ $(3 / 2) \beta$ is sufficient to describe the overall behavior at late times.

Evidence for departures from the simple standard model is provided by, e.g., sharp rises or humps in the light curves followed by a renewed decay, as in GRB $970508([64,66])$. Detailed time-dependent model fits [62] to the X-ray, optical and radio light curves of GRB 970228 and GRB 970508 show that, in order to explain the humps, a non-uniform injection or an anisotropic outflow is required. These fits indicate that the shock physics may be a function of the shock strength (e.g. the electron index $p$, injection fraction $\zeta$ and/or $\epsilon_{b}, \epsilon_{e}$ change in time), and also indicate that dust absorption is needed to simultaneously fit the X-ray and optical fluxes. The effects of beaming (outflow within a limited range of solid angles) can be significant [60], but are coupled with other effects, and a careful analysis is needed to disentangle them.

Prompt optical, X-ray and $\mathrm{GeV}$ flashes from reverse and forward shocks, as well as from internal shocks, have been calculated in theoretical fireball shock models for a number of years $[41,42,57,43,81]$, as have been jets (e.g. [38,40,42], and in more detail $[76,62,60,77])$. However, observational evidence for these effects were largely lacking, until the detection of a prompt (within $22 \mathrm{~s}$ ) optical flash from GRB 990123 with ROTSE by [2], together with X-ray, optical and radio follow-ups $[34,21,18,1,12,27]$. GRB 990123 is so far unique not only for its prompt optical detection, but also by the fact that if it were emitting isotropically, based on its redshift $z=1.6[34,1]$ its energy would be the largest of any GRB so far, $4 \times 10^{54}$ ergs. It is, however, also the first (tentative) case in which there is evidence for jetlike emission $[34,18,12]$. An additional, uncommon feature is that a radio afterglow appeared after only one day, only to disappear the next $[21,34]$.

The prompt optical light curve of GRB 990123 decays initially as $\propto t^{-2.5}$ to $\propto t^{-1.6}[2]$, much steeper than the typical $\propto t^{-1.1}$ of previous optical afterglows detected after several hours. However, after about 10 minutes its decay rate moderates, and appears to join smoothly onto a slower decay rate $\propto t^{-1.1}$ measured with large telescopes $[21,34,18,12]$ after hours and days. The prompt optical flash peaked at 9-th magnitude after $55 \mathrm{~s}[2]$, and in fact a 9-th magnitude prompt flash with a steeper decay rate had been predicted more than two years ago [43], from 
the synchrotron radiation of the reverse shock in GRB afterglows at cosmological redshifts (see also $[81,41,42]$ ). An origin of the optical prompt flash in internal shocks $[43,49]$ cannot be ruled out, but is less likely since the optical light curve and the $\gamma$-rays appear not to correlate well $[82,21]$. The subsequent slower decay agrees with predictions for the forward external shock $[43,82,49]$.

The evidence for a jet is based on an apparent steepening of the light curve after about three days $[34,18,12]$. If real, this steepening is probably due to the transition between early relativistic expansion, when the light-cone is narrower than the jet opening, and the late expansion, when the light-cone has become wider than the jet, leading to a drop in the effective flux $[76,34,49,77]$. A rough estimate leads to a jet opening angle of 3-5 degrees, which would reduce the total energy requirements to about $4 \times 10^{52} \mathrm{ergs}$. This is about two order of magnitude less than the binding energy of a few solar rest masses, which, even allowing for substantial inefficiencies, is compatible with currently favored scenarios (e.g. [68,37]) based on a stellar collapse or a compact merger.

\section{LOCATION AND ENVIRONMENTAL EFFECTS}

The location of the afterglow relative to the host galaxy center can provide clues both for the nature of the progenitor and for the external density encountered by the fireball. A hypernova model would be expected to occur inside a galaxy in a high density environment $n_{o}>10^{3}-10^{5} \mathrm{~cm}^{-3}$. Most of the detected and well identified afterglows are inside the projected image of the host galaxy [6], and some also show evidence for a dense medium at least in front of the afterglow ( [52]).

In NS-NS mergers one would expect a BH plus debris torus system and roughly the same total energy as in a hypernova model, but the mean distance traveled from birth is of order several Kpc [8], leading to a burst presumably in a less dense environment. The fits of [95] to the observational data on GRB 970508 and GRB 971214 in fact suggest external densities in the range of $n_{o}=0.04-0.4 \mathrm{~cm}^{-3}$, which would be more typical of a tenuous interstellar medium. These could be within the volume of the galaxy, but for NS-NS on average one would expect as many GRB inside as outside. This is based on an estimate of the mean NS-NS merger time of $10^{8}$ years. BH-NS mergers would occur in timescales $\sim 10^{7}$ years, and would be expected to give bursts inside the host galaxy ( [8]; see however [19]). In at least one "snapshot" standard afterglow spectral fit for GRB 980329 [75] the deduced external density is $n_{o} \sim 10^{3} \mathrm{~cm}^{-3}$. In some of the other detected afterglows there is other evidence for a relatively dense gaseous environments, as suggested, e.g. by evidence for dust [74] in GRB970508, the absence of an optical afterglow and presence of strong soft X-ray absorption [24,51] in GRB 970828, the lack an an optical afterglow in the (radio-detected) afterglow ( [86]) of GRB980329, and spectral fits to the low energy portion of the X-ray afterglow of several bursts [52]. One important caveat is that all afterglows found so far are based on Beppo-SAX positions, which is sensitive only to long bursts $t_{b} \gtrsim 20 \mathrm{~s}$ [28]. This is significant, 
since it appears likely that NS-NS mergers lead [37] to short bursts with $t_{b} \lesssim 10 \mathrm{~s}$. To make sure that a population of short GRB afterglows is not being missed will probably need to await results from HETE [26] and from the planned Swift [84] mission, which is designed to accurately locate $300 \mathrm{GRB} / \mathrm{yr}$.

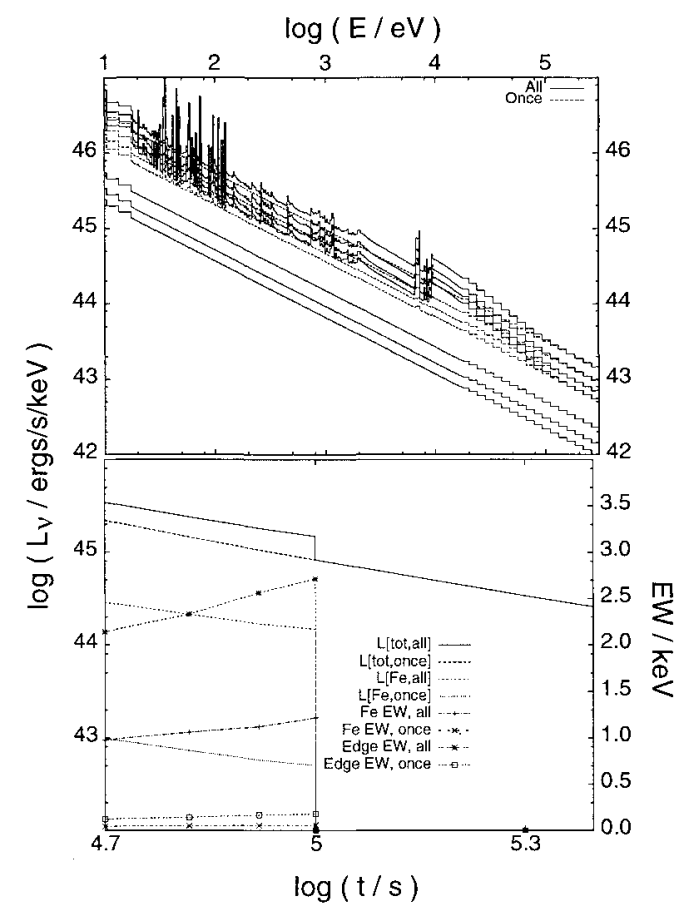

FIGURE 3. Spectrum (top) of a hypernova funnel model for various observer times, showing (bottom) the total and $\mathrm{Fe}$ light curves and equivalent widths (Weth, Mészáros , Kallman \& Rees 1999), for with $R=1.5 \times 10^{16} \mathrm{~cm}$, $n=10^{10} \mathrm{~cm}^{-3}$, and $\mathrm{Fe}$ abundance $10^{2}$ times solar.

The environment in which a GRB occurs may also lead to specific spectral signatures from the external medium imprinted in the continuum, such as atomic edges and lines $[5,65,46]$. These may be used both to diagnose the chemical abundances and the ionization state (or local separation from the burst), as well as serving as potential alternative redshift indicators. (In addition, the outflowing ejecta itself may also contribute blue-shifted edge and line features, especially if metal-rich blobs or filaments are entrained in the flow from the disrupted progenitor debris [45], which could serve as diagnostic for the progenitor composition and outflow Lorentz factor). To distinguish between progenitors, an interesting prediction ( [46]; see also [22,9]) is that the presence of a measurable Fe $\mathrm{K}-\alpha$ X-ray emission line could be a diagnostic of a hypernova, since in this case one may expect a massive envelope at a radius comparable to a light-day where $\tau_{T} \lesssim 1$, capable of reprocessing the X-ray continuum by recombination and fluorescence. Detailed radiative transfer calculations have been performed to simulate the time-dependent X/UV line spectra of massive progenitor (hypernova) remnants [93], see Figure 3. Two groups $[67,98]$ have in fact recently reported the possible detection of Fe emission lines in GRB 970508 and GRB 970828. 
An interesting case is the apparent coincidence of GRB 980425 with the unusual $\mathrm{SN} \mathrm{Ib} / \mathrm{Ic} 1998 \mathrm{bw}[20]$, which may represent a new class of SN [29,7]. If true, this could imply that some or perhaps all GRB could be associated with SN Ib/Ic [90], differring only in their viewing angles relative to a very narrow jet. Alternatively, the GRB could be (e.g. [97]) a new subclass of GRB with lower energy $E_{\gamma} \sim$ $10^{48}\left(\Omega_{j} / 4 \pi\right)$ erg, only rarely observable, while the great majority of the observed GRB would have the energies $E_{\gamma} \sim 10^{54}\left(\Omega_{j} / 4 \pi\right)$ ergs as inferred from high redshift observations. The difficulties are that it would require extreme collimations by factors $10^{-3}-10^{-4}$, and the statistical association is so far not significant [32]. However, two more GRB light curves may have been affected by an anomalous SNR (see, e.g. the review of [94]).

\section{CONCLUSIONS}

The fireball shock model of gamma-ray bursts has proved quite robust in providing a consistent overall interpretation of the major features of these objects at various frequencies and over timescales ranging from the short initial burst to afterglows extending over many months. Significant progress has been made in understanding both the phenomenology and the physics of these obejcts, which may the most widely studied type of black holes sources. There still remain a number of mysteries, especially concerning the identity of their progenitors, the nature of the triggering mechanism, the transport of the energy, the time scales involved, and the nature and effects of beaming. However, the collective theoretical and observational understanding is vigorously advancing, and with dedicated new and planned observational missions under way, further significant progress may be expected in the near future.

I thank M.J. Rees, A. Panaitescu, M. Spada and C. Weth for stimulating collaborations, NASA NAG-5 2857, the Guggenheim Foundation, and the Division of Physics, Math \& Astronomy, Astronomy Visitor and Merle Kingsley funds at Caltech.

\section{REFERENCES}

1. Andersen et al. 1999, Science, 283,2073

2. Akerlof, C., et al., 1999, Nature, 398, 389

3. Beloborodov, A, Stern, B \& Svensson, R (1998) ApJ, 508, L25.

4. Beloborodov, A, astro-ph/9911122

5. Bisnovatyi-Kogan, G \& Timokhin, A, 1997, Astr. Rep. 41, 423

6. Bloom, J., etal, 1998, A\& A Supp.,in press (Procs. Rome Conference on GRB)

7. Bloom, J, et al., 1998, ApJ 506, L105

8. Bloom, J, Sigurdsson, S \& Pols, O, 1999, MNRAS (astro-ph/9805222)

9. Böttcher, M, et al., 1998, astro-ph/9809156 
10. Brainerd, J et al.(1999) in Abstr 19th Texas Symp, Paris (astro-ph/9904039).

11. Brainerd, J. et al.(1998), ApJ, 501:325.

12. Castro-Tirado et al., 1999, Science, 283, 2069

13. Costa, E., et al., 1997, Nature, 387, 783

14. Crider, A. et al.(1997), ApJ, 479:L39.

15. Dermer, C \& Mitman, K, 1998, astro-ph/9809411

16. Dermer, C.D., et al., 1999, Ap.J., 515, L49.

17. Eichler, D \& Levinson, A (1999), ApJ, subm(astro-ph/9903103).

18. Fruchter, A. etal, Ap.J. subm (astro-ph/9902236)

19. Fryer, C \& Woosley, S, 1998, ApJ(Lett) subm (astro-ph/9804167

20. Galama, T. et al., 1998, Nature, 395,670

21. Galama, T. et al., 1999, Nature, 398, 394

22. Ghisellini, G, et al., 1998, astro-ph/9808156

23. Ghisellini, G. and Celotti, A. (1999), ApJ, 511, L93.

24. Groot, P. et al., 1997; in Gamma-Ray Bursts, Meegan, C., Preece, R \& Koshut, T, eds., 1997 (AIP: New York), p. 557

25. Harding, A.K. and Baring, M.G., 1994, in Gamma-ray Bursts, ed. G. Fishman, et al., p. 520 (AIP $307, \mathrm{NY}$ )

26. HETE, http://space.mit.edu/HETE/

27. Hjorth, etal, 1999, Science, 283, 2073

28. Hurley, K., A\& A Supp.,in press (Procs. Rome Conference on GRB)

29. Iwamoto, K, et al., 1998, Nature 395,672

30. Katz, J., 1994a, ApJ, 422, 248

31. Katz, J., 1994b, ApJ, 432, L107

32. Kippen, R.M. et al., 1998, ApJ subm (astro-ph/9806364)

33. Kobayashi, S, Piran, T \& Sari, R, 1998, ApJ, 490, 92

34. Kulkarni, S., et al., 1999, Nature, 398, 389

35. Liang, E et al.(1997), Ap.J., 491, L15.

36. Liang, E. et al.(1999), Ap.J., 519, L21.

37. Macfadyen, A \& Woosley, S, 1999, ApJ in press (astro-ph/9810274)

38. Mészáros, P \& Rees, M.J., 1992, ApJ, 397, 570

39. Mészáros, P. \& Rees, M.J., 1993a, ApJ, 405, 278

40. Mészáros , P., Laguna, P \& Rees, M.J., 1993, ApJ, 415, 181

41. Mészáros, P. and Rees, M.J., 1993b, Ap.J., 418, L59

42. Mészáros, P., Rees, M.J. \& Papathanassiou, H, 1994, Ap.J., 432, 181

43. Mészáros, P \& Rees, M.J., 1997a, ApJ, 476, 232

44. Mészáros, P \& Rees, M.J., 1997b, ApJ, 482, L29

45. Mészáros, P \& Rees, M.J., 1998a, ApJ, 502, L105

46. Mészáros, P \& Rees, M.J., 1998b, MNRAS, 299, L10

47. Mészáros, P, Rees, M.J. \& Wijers, R, 1998, Ap.J., 499, 301 (astro-ph/9709273)

48. Mészáros, P, Rees, M.J \& Wijers, R,1999,New Ast, 4, 313(astro-ph/9808106)

49. Mészáros, P \& Rees, M.J., MNRAS, in press (astro-ph/9902367)

50. Mészáros, P. \& Rees, M.J., ApJ, in press (1999) (astro-ph/9908126).

51. Murakami, T. et al., 1997, in Gamma-Ray Bursts, Meegan, C., Preece, R \& Koshut, T, eds., 1997 (AIP: New York), p. 435 
52. Owen, A., et al, 1998, Astron.\&Astrophys. in press (astro-ph/9809356),

53. Paczyński, B. \& Rhoads, J, 1993, Ap.J., 418, L5

54. Paczyński , B. \& Xu, G., 1994, ApJ, 427, 708

55. Paczyński, B., 1990, Ap.J., 363, 218

56. Paczyński, B., 1998, ApJ, 494, L45

57. Papathanassiou, H \& Mészáros, P, 1996, ApJ, 471, L91

58. Panaitescu, A \& Mészáros, P, 1998a, ApJ, 492, 683

59. Panaitescu, A. \& Mészáros, P., 1998b, ApJ, 501, 772

60. Panaitescu, A. \& Mészáros, P., 1999, ApJ, in press (astro-ph/9806016)

61. Panaitescu, A. \& Mészáros, P., 1998d, ApJ, subm (astro-ph/9810258)

62. Panaitescu, A, Mészáros, P \& Rees, MJ, 1998, ApJ, 503, 314

63. Panaitescu, A., Spada, M., and Mészáros , P. (1999), ApJ, 522:L105.

64. Pedersen, H. et al.(1998), ApJ 496, 311

65. Perna, R. \& Loeb, A., 1998, ApJ, 503, L135

66. Piro, L, et al., 1998, A \& A, 331, L41

67. Piro, L, et al., 1998b, A\& A Supp.,in press (Procs. Rome Conference on GRB)

68. Popham, R., Woosley, S \& Fryer, C., 1998, ApJ n press (astro-ph/9807028)

69. Preece, R. et al.(1998), ApJ, 496, 849.

70. Rees, M.J. \& Mészáros, P., 1992, MNRAS, 258, P41

71. Rees, M.J. \& Mészáros, P., 1994, Ap.J, 430, L93

72. Rees, M.J. \& Mészáros, P., 1998, ApJ, 496, L1

73. Reichart, D., 1997, ApJ, 485, L57

74. Reichart, D., 1998, ApJ, 495, L99

75. Reichart, D \& Lamb, D.Q, 1998, A\&A Supp,in press (Procs. Rome Conf)

76. Rhoads, J, 1997, Ap.J., 487, L1

77. Rhoads, J, 1999, ApJ subm, astro-ph/9903383

78. Sari, R. \& Piran, T., 1995, ApJ, 455, L143

79. Sari, R \& Piran, T, 1998, ApJ, 485, 270

80. Sari, R, Piran, T \& Narayan, R, 1998, ApJ, 497, L17 (astro-ph/9712005)

81. Sari, R \& Piran, T., 1999a, A\&A subm (astro-ph/9901105)

82. Sari, R \& Piran, T, 1999b, Ap.J. subm (astro-ph/9902009)

83. Spada, M, Panaitescu, A \& Mészáros, P, 1999 ApJ subm(astro-ph/9908097)

84. Swift, http://swift.gsfc.nasa.gov/

85. Tavani, M., 1997, ApJ, 483, L87

86. Taylor, G.B., et al., 1997, Nature, 389, 263

87. Thompson, C., 1994, MNRAS, 270, 480

88. van Paradijs, J, et al., 1997, Nature,386, 686

89. Vietri, M., 1997a, ApJ, 478, L9

90. Wang, L. \& Wheeler, J.C., 1998, ApJ subm (astro-ph/9806212)

91. Waxman, E., 1997, ApJ, 485, L5

92. Waxman, E., 1997b, ApJ, 489, L33

93. Weth, C, Mészáros , P, Kallman, T \& Rees, M.J, 1999 ApJ in press(astro$\mathrm{ph} / 9908243)$

94. Wheeler, J.C. (1999), in Supernovae 8 Gamma Ray Bursts, eds. M Livio et al.(Cambridge U.P.)(astro-ph/9909096). 
95. Wijers, R.A.M.J. \& Galama, T., 1998, ApJ, in press (astro-ph/9805341)

96. Wijers, R.A.M.J., Rees, M.J. \& Mészáros, P., 1997, MNRAS, 288, L51

97. Woosley, S., Eastman, R. \& Schmidt, B., 1998, ApJ, subm (astro-ph/9806299)

98. Yoshida, A, et al., 1998, A\& A Supp.,in press (Procs. Rome Conf on GRB) 